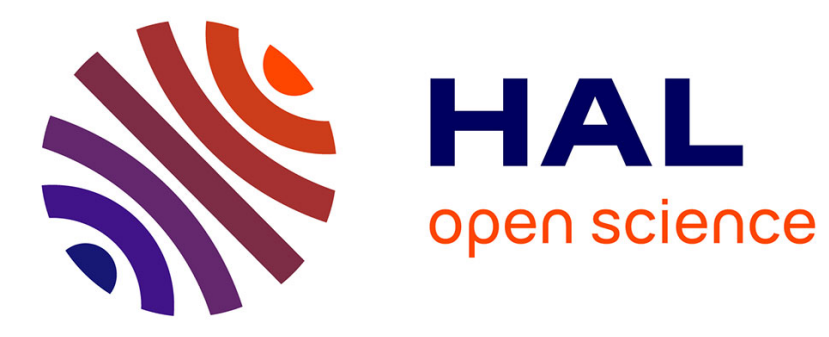

\title{
Quaternion-based IMU and stochastic error modeling for intelligent vehicles
}

Thomas Brunner, Jean-Philippe Lauffenburger, Sébastien Changey, Michel Basset

\section{- To cite this version:}

Thomas Brunner, Jean-Philippe Lauffenburger, Sébastien Changey, Michel Basset. Quaternion-based IMU and stochastic error modeling for intelligent vehicles. 2015 IEEE Intelligent Vehicles Symposium (IV), IEEE, Jun 2015, Seoul, South Korea. pp.877-882, 10.1109/IVS.2015.7225795 hal-01331721

\section{HAL Id: hal-01331721 \\ https://hal.science/hal-01331721}

Submitted on 14 Jun 2016

HAL is a multi-disciplinary open access archive for the deposit and dissemination of scientific research documents, whether they are published or not. The documents may come from teaching and research institutions in France or abroad, or from public or private research centers.
L'archive ouverte pluridisciplinaire HAL, est destinée au dépôt et à la diffusion de documents scientifiques de niveau recherche, publiés ou non, émanant des établissements d'enseignement et de recherche français ou étrangers, des laboratoires publics ou privés. 


\title{
Quaternion-based IMU and stochastic error modeling for intelligent vehicles
}

\author{
Thomas Brunner ${ }^{1}$, Jean-Philippe Lauffenburger ${ }^{2}$, Sébastien Changey ${ }^{1}$ and Michel Basset ${ }^{2}$
}

\begin{abstract}
This paper focuses on the development of an IMU measurement simulator for navigation estimation algorithms validation. Its aim is to generate the sensor measurements thanks to an input trajectory described by the position and the orientation. The proposed models are derived from an inverse kinematic modeling of the sensors and an identification of their stochastic errors. These latter are composed of the biases instability, random walks and finally the sensors dynamics and bandwidth. The error model parameters of a low cost MEMSIMU are determined using the Allan Variance method. In a second step, a Matlab simulator is built gathering the aforementioned models. Thanks to their completeness, this simulation tool is characterized by its wide range of application fields and dynamics that can be described. Its aim is to determine, from the time-dependent position and orientation data, the IMU measurements (3D accelerations and angular rates) without any object model. Finally, the simulator is validated using real experiments performed with an instrumented test car in normal driving as well as in obstacle avoidance situations.
\end{abstract}

\section{INTRODUCTION}

Dynamic location of a moving object is a predominant research topic for intelligent and autonomous vehicles. In general, GNSS (Global Navigation Satellite System) and Inertial Measurement Units (IMUs) are combined to achieve better precision and integrity. Unfortunately, in urban and suburban areas, the satellite signals suffer from poor quality, multipath or outages, leaving the location algorithms with the inertial data only. In these non-optimal conditions, multiple solutions are investigated to perform accurate location: 3D map-aided location [1] to forecast the visibility of the satellites, vision-only positioning [2] or SLAM-based methods [3]. Also, vehicle-to-infrastructure (V2I) or vehicle-tovehicle $(\mathrm{V} 2 \mathrm{~V})$ communication using VANETs can provide additional data for vehicle positioning [4].

Considering GPS/INS integrated positioning solutions, the performance during GPS dysfunctions are closely related to the drift and IMU sensor errors. When not modeled properly or not correctly considered in the positioning algorithms, these drifts can lead to strong position errors. This paper focuses on the latter mentioned aspect. The retained approach to improve location is the use of multiple low cost IMUs. The final aim (out of the scope of this paper) is to develop multiple IMU algorithms that can achieve good performance

\footnotetext{
*This work was supported by the Alsace Region and the French-German Research Institute of Saint-Louis.

${ }^{1}$ Thomas Brunner and Sbastien Changey are with French-German Research Institute of Saint-Louis (ISL), Guidance, Navigation and Control (GNC) department, Saint-Louis, France thomas.brunnerdisl .eu

${ }^{2}$ Jean-Philippe Lauffenburger and Michel Basset are with Laboratoire MIPS-EA2332, Université de Haute Alsace, Mulhouse, France jean-philippe. lauffenburgereuha.fr
}

with low cost redundant sensors. With the rapid development of the MEMS (Micro-Electro-Mechanical Systems) technology, it is now possible to deploy multiple IMUs in a vehicle. Exploiting redundancy and information contained in the signal differences can extend the positioning performance significantly [5]. Moreover, inertial data provides information on the current states of the car: orientation, slip angle, etc. Those variables are crucial for vehicle control applications.

In a first step, a precise modeling of the IMU sensors and especially their errors is necessary. That is why, kinematic models of the sensors and stochastic error models are developed. The typical imperfections modeled are the sensor dynamics, constant and dynamic biases and white noises. Their parameters are identified following the temporal Allan Variance technique [6]. The kinematic modeling is derived providing the sensors (rate gyroscopes and accelerometers) outputs with respect to the given trajectory data (position and orientation of the vehicle). In a second step, a Matlab simulator gathering these models is developed. It is dedicated to a wide range of application fields (including road and air transportation systems as well as high dynamic flying systems). Due to these objectives, it appears clearly that an application-dependent system model generally used to enhance the location precision cannot be considered here. Moreover to be robust to these constraints, the quaternions are selected to represent the object orientation. Indeed, quaternions do not suffer from the angle singularities known as the gimbal lock problem. The sensor data simulator has already been validated with real measurements considering low dynamics [7], [8]. In this paper, a validation is proposed in the intelligent vehicles context using low and high dynamics issued from real trials.

Considering the simulation of sensors, existing simulators have different focus. For example, in [9], the focus is on the error models for the skewed sensors, the measurements are not determined from an input trajectory. In [10], a simulator is built for different applications: to perform and test calibration processes and to validate new mathematical models involving IMUs. Finally, if quaternions have already been used in GPS/INS navigation algorithms [11], their use in sensor measurment simulations without system models is still marginal. Unfortunately, the validation process does not fit our application field.

The outline of the paper is as follows. Section II describes the theoretical background. Section III is devoted to the description of the sensor measurements and the inverse kinematic models needed to determine the sensor data. Section IV refers to the error models identification. Results are shown 


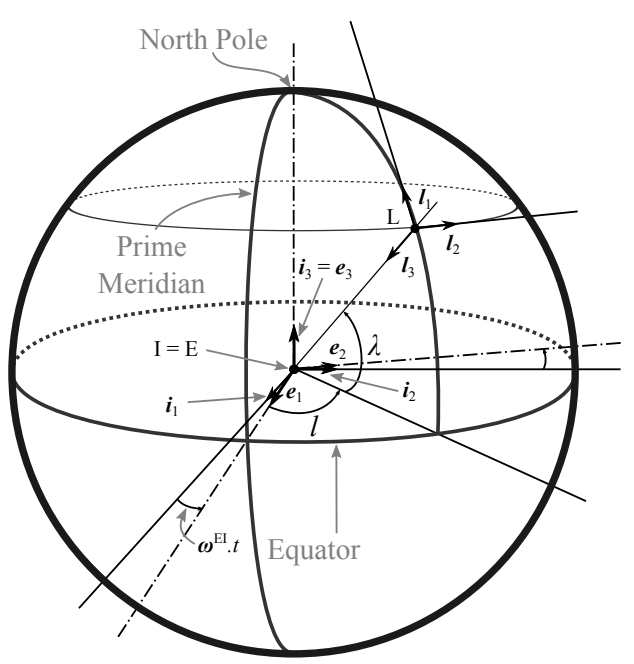

Fig. 1. The inertial frame (I), the earth frame (E) and the local coordinate system (L).

in section V. In this section, different test scenarios are described and performed using an instrumented test car. Section VI concludes the paper.

\section{THEORETICAL BACKGROUND}

\section{A. Reference Frames}

Reference frames and coordinate systems are widely used in the navigation field ([12] and [13]) to express correctly all the information. To help the reader to understand this paper, a short description of each frame is given.

- The Inertial frame (I) is centered on the Earth. Its third axis is directed to the North Pole. The others axes are in the equator plan. This frame is not rotating with the Earth, for this reason it is considered as the inertial reference.

- The Earth frame (E) which is centered on the Earth, has the same axes than the Inertial frame (I) at $t=0$. Unlike (I), (E) is following the Earth rotation with a constant speed noted $\omega^{\mathrm{EI}}$.

- The Local coordinate system (L) is located at the center of gravity of the considered object. Its orientation is tangent to the surface of the Earth and defined with its position with respect to (E) by 2 angles: the latitude $(\lambda)$ and the longitude $(l)$.

- The Body frame (B) is linked to the object orientation. Its orientation with respect to $(\mathrm{L})$ is given by the classical Euler angles.

- The IMU frame (W), attached to the sensors, can be misaligned with the body frame. Its position and orientation depend on the hardware configuration of the IMU with respect to the object.

The Inertial and Earth frames and the Local coordinate system are illustrated in figure 1. For clarity reason, the body and the work frames are not illustrated. Any axis of a frame is named by the lowercase of the frame name and a subscript giving its number such that the 3 vectors of the frame $(\mathrm{E})$ are $e_{1}, e_{2}$ and $e_{3}$ (see figure 1 ).

\section{B. Quaternions}

There are multiple ways to represent the orientation of one frame with respect to another. The most known is the Euler angle system, but it suffers from gimbal lock. This problem gives numerical issues for certain known orientations. To avoid this limitation in the current work, quaternions [14] are retained for the sensor measurement modeling. This section gives the necessary background related to quaternions. Readers willing to have details can refer to [15].

Introduced by Hamilton, quaternions are hypercomplexe numbers with 4 components: a scalar $q_{0}$ and one vector $q_{\mathrm{v}}$ composed by 3 imaginary numbers $q_{1}, q_{2}$ and $q_{3}$. One quaternion $q$ can be expressed as

$$
q=\left\{\begin{array}{l}
q_{0} \\
q_{\mathrm{v}}
\end{array}\right\}
$$

or

$$
q=q_{0}+q_{1} i+q_{2} j+q_{3} k
$$

with vectors $i, j$ and $k$ describing an orthogonal basis. A pure quaternion is composed of a scalar part $q_{0}$ equal to 0 . To avoid confusion between vectors and quaternions, brackets \{ \} are used instead of [ ].

The multiplication between 2 quaternions, written $r=p \otimes$ $q$, is obtained by

$$
r=\left\{\begin{array}{c}
p_{0} q_{0}-p_{\mathrm{v}} \cdot q_{\mathrm{v}} \\
p_{0} q_{\mathrm{v}}+q_{0} p_{\mathrm{v}}+p_{\mathrm{v}} \wedge q_{\mathrm{v}}
\end{array}\right\} .
$$

Rotations with quaternions: The complex part of the quaternion $\left(q_{\mathrm{v}}\right)$ is equivalent to a vector. This is used to represent rotations with quaternions: a rotation around an axis $d$ by an angle $\alpha$ can be represented by a unit quaternion (its norm is equal to 1) [16]

$$
q=\underbrace{\cos \frac{\alpha}{2}}_{q_{0}}+\underbrace{\sin \frac{\alpha}{2} d}_{q_{v}} .
$$

The quaternion can express any 3-D rotation since any combination of rotations can be resumed by one unique rotation. The image $x^{\prime}$ of a vector $x$ by the rotation expressed by $q$, can be obtained by the following equation

$$
x^{\prime}=q \otimes x \otimes \tilde{q},
$$

where $\tilde{q}$, the conjugate of $q$ is defined as $\tilde{q}=q_{0}-q_{\mathrm{v}}$. In equation 5 , the vectors $x$ and $x^{\prime}$ are considered as pure quaternions.

\section{SENSORS MODELING}

In this section, the error-less equations of the sensor measurements are derived. The sensor outputs are defined and expressed as a function of the object trajectory, input of the simulator. An inverse kinematic model defines the sensor outputs from the trajectory and orientation of the vehicle as illustrated in figure 2 . From a trajectory describing the evolution of the spatial position $\left[s_{\mathrm{BL}}\right]^{\mathrm{L}}$ and the angular position of the object with respect to a frame $\mathrm{L}$ (using the quaternion $\left\{q^{\mathrm{BL}}\right\}^{\mathrm{L}}$ ), the accelerations and the rotational speeds of the IMU sensors in the frame $\mathrm{W}$ are defined. 


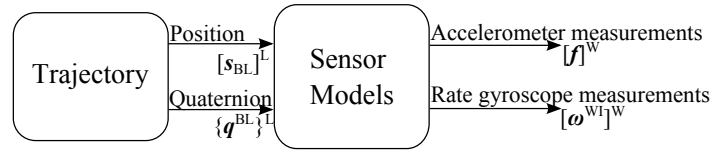

Fig. 2. Inverse kinematic model.

\section{A. Rate Gyroscopes Modeling}

The rate gyroscope output is the angular rate of the sensor with respect to the inertial frame [14], expressed in the sensors frame $(\mathrm{W})$. To determine the rotational speed, the evolution equation of the quaternion is used [15]

$$
\left\{\dot{q}^{\mathrm{WI}}\right\}^{\mathrm{I}}=\frac{1}{2}\left\{q^{\mathrm{WI}}\right\}^{\mathrm{I}} \otimes\left\{\omega^{\mathrm{WI}}\right\}^{\mathrm{W}},
$$

with $\left\{q^{\mathrm{WI}}\right\}^{\mathrm{I}}$ the quaternion expressing the orientation of $(\mathrm{W})$ in (I), $\left\{\dot{q}^{\mathrm{WI}}\right\}^{\mathrm{I}}$ its derivative with respect to time and $\left\{\omega^{\mathrm{WI}}\right\}^{\mathrm{W}}$ the pure quaternion formed from the rotational speed vector of (W) with respect to (I). From (6), it is straightforward to obtain the angular rate sensor output vector $\left\{\omega^{\mathrm{WI}}\right\}^{\mathrm{W}}$

$$
\left\{\omega^{\mathrm{WI}}\right\}^{\mathrm{W}}=2\left\{\tilde{q}^{\mathrm{WI}}\right\}^{\mathrm{I}} \otimes\left\{\dot{q}^{\mathrm{WI}}\right\}^{\mathrm{I}} .
$$

\section{B. Accelerometers Modeling}

The output of an accelerometer is the specific force applied on the sensor along its sensitive axis (in frame $\mathrm{W}$ ). The specific force is defined by the sum of all non-gravitational forces applied on the object divided by the object mass. It is then the absolute acceleration of the object $\left(a_{\mathrm{W}}^{\mathrm{I}}\right)$ minus the gravitational field, such that

$$
[f]^{\mathrm{W}}=\left[a_{\mathrm{W}}^{\mathrm{I}}\right]^{\mathrm{W}}-\left[g_{\mathrm{f}}\right]^{\mathrm{W}} .
$$

From [13], the gravitational field expression is

$$
g_{\mathrm{f}}=g+\gamma_{e}=-G M \frac{s_{\mathrm{WE}}}{\left\|s_{\mathrm{WE}}\right\|^{3}}-\omega^{\mathrm{EI}} \wedge\left(\omega^{\mathrm{EI}} \wedge s_{\mathrm{WE}}\right),
$$

where $s_{\mathrm{WE}}$ is the position of the sensors with respect to (E), $G$ is the gravitational constant and $M$ is the mass of the Earth.

The objective is then to determine the expression of $[f]^{\mathrm{W}}$ from the evolution of the object position $\left[s_{\mathrm{BL}}\right]^{\mathrm{L}}$ which is an input of the simulator. However, the sensors are not positioned at the center of the object. Consequently, deriving the position of the object will not lead to the acceleration of the IMU but to the object one. After transforming the input position $\left[s_{\mathrm{BL}}\right]^{\mathrm{L}}$ in frame $(\mathrm{E})$, the total acceleration applied on the IMU is given by ${ }^{1}[13]$

$$
\begin{aligned}
a_{\mathrm{W}}^{\mathrm{I}}= & \overbrace{a_{\mathrm{B}}^{\mathrm{E}}}^{\mathrm{I}}+\overbrace{\left.\frac{d \omega^{\mathrm{BE}}}{d t}\right|_{\mathrm{B}} \wedge s_{\mathrm{WB}}+\omega^{\mathrm{BE}} \wedge\left(\omega^{\mathrm{BE}} \wedge s_{\mathrm{WB}}\right)}^{\mathrm{II}} \\
& +\underbrace{\omega^{\mathrm{EI}} \wedge\left(2\left(v_{\mathrm{B}}^{\mathrm{E}}+\omega^{\mathrm{BE}} \wedge s_{\mathrm{WB}}\right)+\omega^{\mathrm{EI}} \wedge s_{\mathrm{WI}}\right)}_{\text {III }},(10)
\end{aligned}
$$

\footnotetext{
${ }^{1}$ For the sake of clarity, it's assumed that all variables are expressed in the same coordinate system, the brackets []$^{\mathrm{X}}$ are then forsaken.
}

where I represents the acceleration of the object with respect to the Earth, II is the acceleration due to the position difference between the IMU and the object center of gravity and III is the acceleration involved by the Earth rotation around its axis. Even if this latter part can be dismissed in the context of intelligent vehicles, it is necessary to take it into account in other kind of application fields. Finally, the specific force can be obtained by subtracting (9) from (10).

\section{Stochastic Sensor ERror Modeling}

\section{A. Model Description}

Generally, stochastic error models are 5-term polynomials as described in [17]. For the development of the current application, the focus is made on 3 main terms: the bias instability, the rate random walk and the angular random walk. In addition, the sensor dynamics is also considered.

1) Sensor Dynamics and Bandwidth: One of the main limitations of the sensor performance is its dynamic behavior. To describe it, a first order transfer function is used to model the bandwidth.

$$
K(s)=\frac{\omega_{c}}{s+\omega_{c}},
$$

with $\omega_{c}$ a configuration parameter of the simulator defining the cut-off pulsation at $-3 \mathrm{~dB}$ which can be read on the sensor datasheet. It is a user-defined parameter in the simulator.

2) Bias Modeling: Modeling the bias implies the description of a more complex model than a simple constant. Indeed, the bias has a time evolving part $\left(b_{\mathrm{ev}}\right)$ in addition of the constant part $\left(b_{\mathrm{cst}}\right)$. To model the bias evolution, two processes are used, as suggested in [18]; a first order Gauss Markov process $\left(b_{\mathrm{gm}}\right)$ and a random walk $\left(b_{\mathrm{rw}}\right)$. The first is used to represent a wide number of physical processes. It is an autoregressive process with a correlation time $T_{c}$. The parameters needed are the correlation time and the standard deviation $\sigma_{\mathrm{gm}}$ of the noise. The random walk, in the other hand, is the result of the integration of a white noise. Finally, the evolving part of the bias is given by

$$
\begin{aligned}
{\left[\begin{array}{c}
b_{\mathrm{gm}} \\
b_{\mathrm{rw}}
\end{array}\right]_{k}=} & {\left[\begin{array}{cc}
\left(1-\frac{\Delta t}{T_{c}}\right) & 0 \\
0 & 1
\end{array}\right]\left[\begin{array}{l}
b_{\mathrm{gm}} \\
b_{\mathrm{rw}}
\end{array}\right]_{k-1} } \\
& +\left[\begin{array}{c}
\sigma_{\mathrm{gm}} \sqrt{\left(1-\exp \left(-2 \frac{\Delta t}{T_{c}}\right)\right)} \\
\sigma_{\mathrm{rw}} \sqrt{\Delta t}
\end{array}\right] u_{k}, \\
b_{\mathrm{ev}}= & {\left[\begin{array}{ll}
1 & 1
\end{array}\right]\left[\begin{array}{c}
b_{\mathrm{gm}} \\
b_{\mathrm{rw}}
\end{array}\right]_{k}, }
\end{aligned}
$$

with $u_{k}$ a unitary white noise.

The last implemented process error is $w$, the error due to the white noise. This noise is considered as zero-mean and Gaussian. The last parameter to be specified is then its standard deviation.

\section{B. Stochastic Parameter Identification}

To simulate a commercially available low-cost IMU, multiple experiments were conducted on a Xsens MTI MEMSIMU [19]. The Allan Variance has been employed as suggested by [6] and [20] to identify the noise parameters. It is a widely used time-domain technique allowing determining 


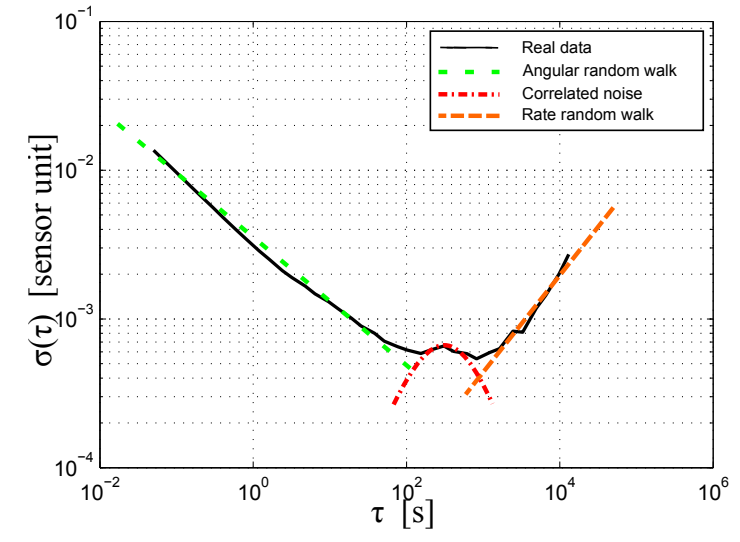

Fig. 3. Application of the Allan Variance on one accelerometer.

long-term noises. To perform this approach, the IMU has to remain completely still during several hours. Figure 3 shows a typical Allan Variance plot and its interpretation, applied on real measurements. The values are extracted from the measurements as explained in [20]. The hypothetical Allan Variance sketches (the dotted lines) are the typical noise components. The angular random walk slope illustrates the effect of a white noise. The rate random walk slope is the result of a random walk noise (cf. section IV-A) and the correlation noise slope comes from the correlated part of the measurements. As the variance is a function of the time cluster size $(\tau)$, it is possible to interpret the influence of each type of noise: the angular random walk noise is a problem in short time but can be reduced by averaging the measurements while the rate random walk noise is more affecting the long term measurements. Table I shows the results of this procedure for the Xsens MTI IMU [19] mounted in the test car (see section V). It gathers all the needed data for the simulation of the IMU imperfections modeling and sensor data simulation. The results of the performed Allan Variance are in correlation with the sensor datasheets.

\section{Results}

In this section, different test scenarios with different dynamics are employed to validate the stochastic sensor modeling. These scenarios describe the evolution of a driving car. The first tests are standard vehicle dynamic tests usually performed by car manufacturers to evaluate the car's dynamics under lateral solicitations. One test is conducted under constant lateral acceleration (curve at constant longitudinal velocity) whereas the second trial is a standard ISO lane change maneuver (moose test). These trajectories are performed with an instrumented Renault Scenic 1 of MIPS laboratory. The last scenario is a longer trajectory with the car driving normally around the university campus. This test is done thanks to the Renault Scenic 3 shown in figure 4.

\section{A. Lateral Dynamics Validation}

1) High Grip Bend Maneuver: The first test is a high grip bend maneuver. The car reaches a cruise speed of $50 \mathrm{~km} / \mathrm{h}$, and performs a left turn with a constant curvature

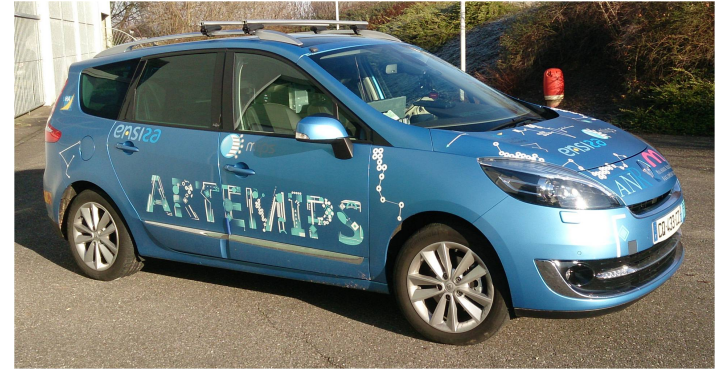

Fig. 4. Test car for the normal driving part.

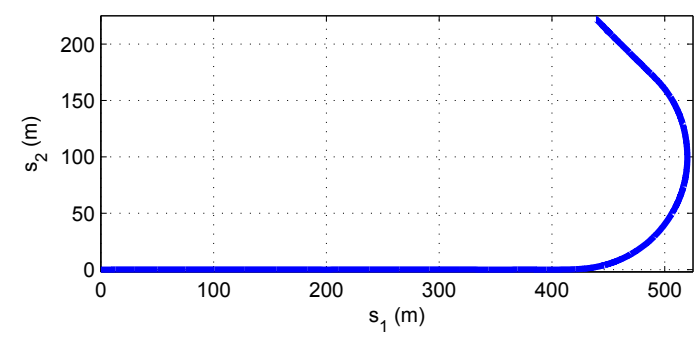

Fig. 5. Trajectory of the vehicle during maneuver.

radius. The GPS measured trajectory is shown in figure 5 and used as the simulator input trajectory. The simulated sensor measurements can be directly compared to the real IMU measurements. The results on the accelerometers and rate gyroscopes are illustrated in figure 6 and 7 .

Figure 6 shows the accelerations measured by the embedded IMU and its corresponding simulations. During the turn, a constant acceleration of $-2 \mathrm{~m} / \mathrm{s}^{2}$ can be observed on the lateral axis. The first axis $\left(f_{1}\right)$ shows the longitudinal acceleration due to the changes in the car speed. It can be seen that the transitory and stationary parts of the accelerations are well reproduced by the simulator.

In this trajectory, the car changes orientation only during the constant curvature radius turn. This means the only rotational speed observed is on the yaw axis, as it can be seen in figure 7 . As well as the accelerations, the simulation
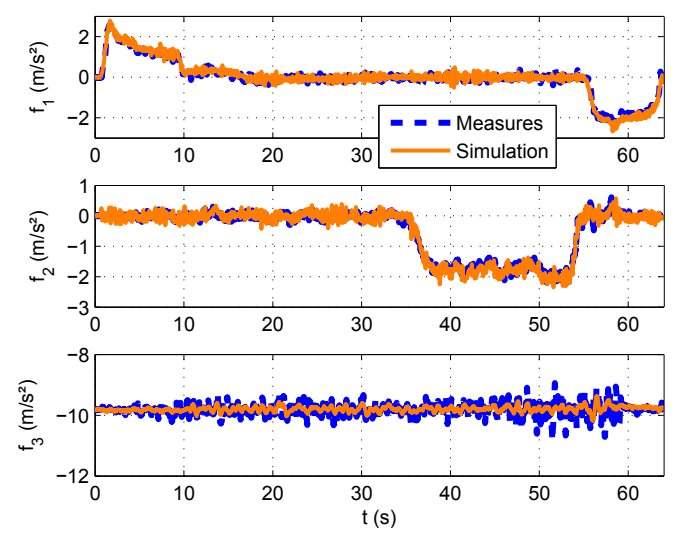

Fig. 6. Accelerometer measurements during the high grip bend test. 


\begin{tabular}{cccc|ccc}
\hline \hline & \multicolumn{3}{c|}{ Accelerometers $\left(\right.$ unit $\left.=m / s^{2}\right)$} & \multicolumn{2}{c}{ Rate Gyroscopes $\left(\right.$ unit $\left.={ }^{\circ} / \mathrm{s}\right)$} \\
\cline { 2 - 7 } & $f_{1}$ & $f_{2}$ & $f_{3}$ & $\omega_{1}$ & $\omega_{2}$ & $\omega_{3}$ \\
\hline \hline$\sigma_{\mathrm{wn}}\left(\right.$ unit $\left./ \sqrt{H_{z}}\right)$ & $1.0 \cdot 10^{-3}$ & $1.2 \cdot 10^{-3}$ & $1.3 \cdot 10^{-3}$ & $4.3 \cdot 10^{-2}$ & $4.5 \cdot 10^{-2}$ & $5.2 \cdot 10^{-2}$ \\
\hline$\sigma_{\mathrm{rw}}\left(\right.$ unit $\left.\cdot \sqrt{H_{z}}\right)$ & $5.4 \cdot 10^{-6}$ & $6.3 \cdot 10^{-6}$ & $6.2 \cdot 10^{-6}$ & $1.1 \cdot 10^{-3}$ & $2.2 \cdot 10^{-4}$ & $1.9 \cdot 10^{-4}$ \\
\hline$\sigma_{\mathrm{gm}}($ unit $)$ & $7.4 \cdot 10^{-4}$ & $7.9 \cdot 10^{-4}$ & $3.4 \cdot 10^{-4}$ & $8.0 \cdot 10^{-2}$ & $1.2 \cdot 10^{-2}$ & $1.5 \cdot 10^{-2}$ \\
\hline$\tau_{\mathrm{gm}}(s)$ & 80 & 10 & 100 & 400 & 300 & 100 \\
\hline \hline
\end{tabular}

TABLE I

ALLAN VARIANCE ERROR IDENTIFICATION RESUlts OF THE LOW-COST XSENS MTI [19].
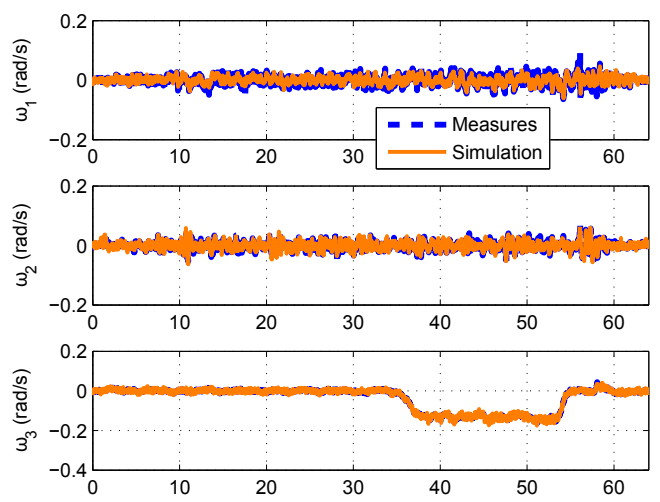

Fig. 7. Rate gyroscope measurements during the high grip bend test.

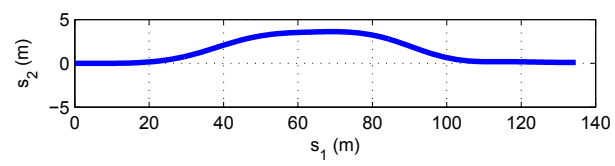

Fig. 8. Trajectory of the vehicle during the obstacle avoidance test.

reproduce correctly the rate gyroscopes measurements during the transitory and the stationary parts of the trajectory.

2) Moose Test: This normalized test simulates the avoidance of a Moose appearing suddenly on the road. Its trajectory is shown in figure 8 . For this test, the car speed during the evasive maneuver was near $110 \mathrm{~km} / \mathrm{h}$. The results on the accelerometer and rate gyroscope measurements are illustrated respectively in figure 9 and 10.

The first plot in figure 9 describes the longitudinal acceleration of the car. A first acaelration phase is done to reach the desired speed of $110 \mathrm{~km} / \mathrm{h}$. The avoidance maneuver begins at $t=25 s$ (see $f_{2}$ plot representing the transversal solicitations). At this time, a transverse acceleration can be observed on the second axis. As the test was performed on a flat surface, the vibrations shown on the last plot are due to the road imperfections. It can be seen that the estimated measurements from the models are very close to the real sensor measurements. In figure 10, the rate gyroscope measurements are plotted. The angular rates are well reproduced, even the roll $\left(\omega_{1}\right)$ and the pitch $\left(\omega_{2}\right)$ rates. This trajectory allows testing the simulator with higher dynamics than the previous one.
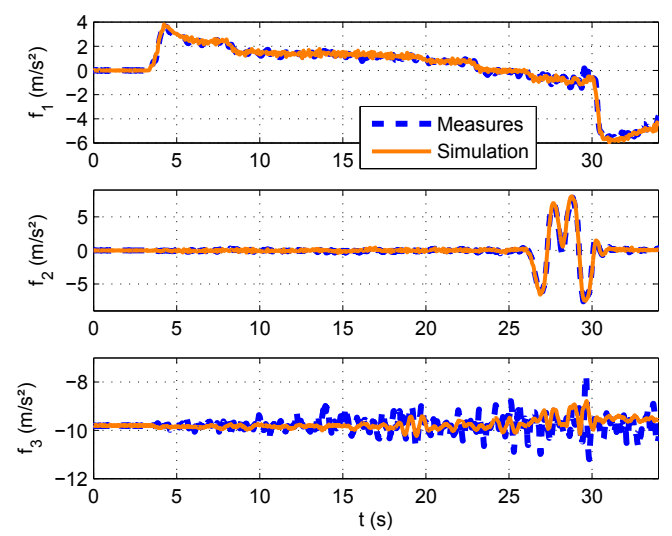

Fig. 9. Accelerometer measurements during the moose test.
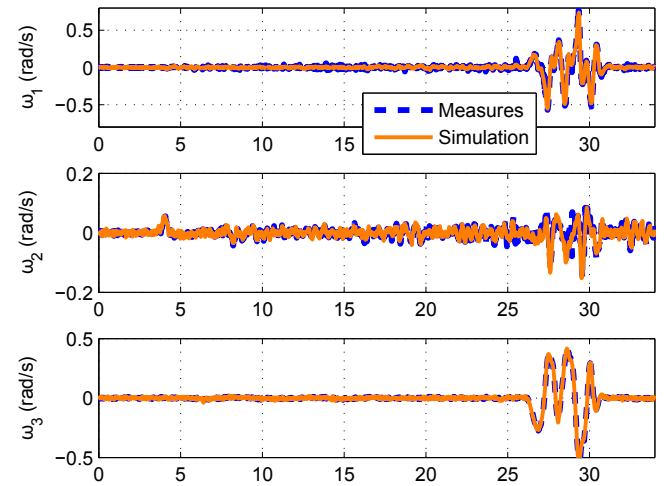

Fig. 10. Rate gyroscope measurements during the moose test.

\section{B. Normal Driving}

The last test correpsonds to a normal and longer path around the university campus. This campus is approximately $800 \mathrm{~m}$ long, $300 \mathrm{~m}$ wide and has an altitude difference of $43 \mathrm{~m}$. The complete vehicle trajectory is 7-minutes long. After driving around the university campus, multiple 8-shape turns on the central path of the campus have been done. This trajectory can be observed in figure 11. The acceleration data are shown in figure 12. It can be observed that the simulated accelerations fit well the shape of the measurements for the longitudinal and transversal accelerations. Concerning the vertical acceleration, the simulated data is very flat compared to the corresponding measurement. This is mainly due to the fact that the road quality is bad around the campus (especially between $0 \mathrm{~s}$ to $50 \mathrm{~s}$, and $350 \mathrm{~s}$ to $380 \mathrm{~s}$ ) leading to numerous 


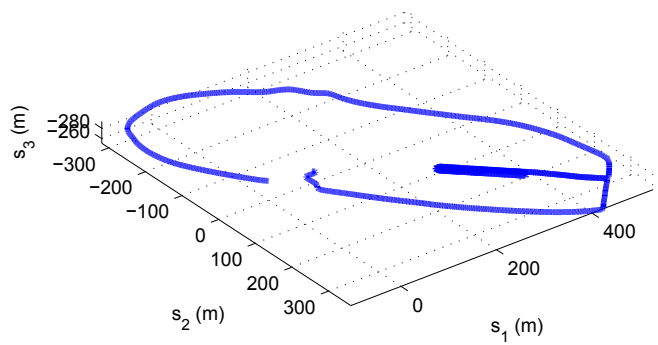

Fig. 11. Trajectory of the vehicle during a campus tour
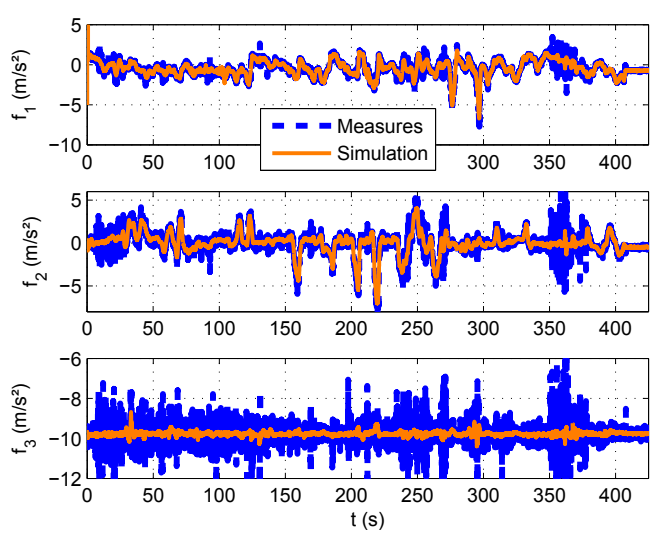

Fig. 12. Accelerometer measurements during the campus tour.

high frequency data on the vertical accelerometer but also the longitudinal and lateral ones. Obviously, these elements cannot be given by the simulator based on the GPS path.

\section{CONCLUSions}

In this paper, an accurate kinematic model has been developed to express IMU sensor (accelerometers and rate gyroscopes) outputs for a given trajectory. The sensor measurements are determined based on the position and orientation of the object considered with respect to the local frame. In addition, in order to provide highly realistic data, stochastic error models of the sensors are added. They are composed of biases, white noises and rate random walk processes. The imperfection parameters have been identified thanks to the Allan Variance technique. Finally, the bandwidth of the sensors is modeled through a first order system representing the sensor dynamics. A Matlab simulator has been developed using this inverse sensor model. Thanks to the modeling efforts, the simulator is valid for a wide range of application fields from low to very high dynamics, short and long range paths, etc. This tool is able to precisely compute the measurements of an IMU for a given input trajectory, described by the $3 \mathrm{D}$ position and orientation of the considered object. A validation process has been conducted. Real experiments have been performed with an instrumented test car in different situations: normal driving and obstacle avoidance scenarios.

Future investigations will be devoted to the extension of the simulation tool in order to estimate the vehicle slip angle. Finally, a real-time implementation of the IMU simulator would allow performing fault detection and diagnosis on IMU measurements.

\section{REFERENCES}

[1] S. Peyraud, D. Bétaille, S. Renault, M. Ortiz, F. Mougel, D. Meizel, and F. Peyret, "About non-line-of-sight satellite detection and exclusion in a 3d map-aided localization algorithm," Sensors, pp. 829-847, Jan. 2013.

[2] H. Lategahn and C. Stiller, "Vision-only localization," IEEE Transaction on Intelligent Transportation Systems, pp. 1246-1257, June 2014.

[3] B. Clarke, S. Worrall, G. Brooker, and E. Nebot, "Towards mapping of dynamic environments with fmcw radar," in Intelligent Vehicles Symposium, (Gold Coast, Australia), pp. 147-152, IEEE, June 2013.

[4] U. A. Peker, T. Acarman, C. Yaman, and E. Yuksel, "Vehicle localization enhancement with vanets," in Intelligent Vehicules Symposium, (Dearborn, Michigan, USA), pp. 661-666, IEEE, June 2014.

[5] D. Bittner, J. Christian, R. Bishop, and D. May, "Fault detection, isolation, and recovery techniques for large clusters of inertial measurement units," in Position, Location and Navigation Symposium, (Monterey, CA), pp. 219-229, IEEE/ION, May 2014.

[6] Y. Zhao, M. Horemuz, and L. E. Sjöberg, "Stochastic modeling and analysis of imu sensor errors," Photogrammetry, Cartography and Remote Sensing, vol. 22, pp. 437-449, 2011.

[7] T. Brunner, S. Changey, E. Pecheur, J.-P. Lauffenburger, and M. Basset, "Evaluation of attitude estimation algorithms using absolute magnetic reference data: Methodology and results," in Position, Location and Navigation Symposium, (Monterey, CA), pp. 212-218, IEEE/ION, May 2014.

[8] T. Brunner, J.-P. Lauffenburger, S. Changey, and M. Basset, "Magnetometer-augmented imu simulator: in-depth elaboration," Sensors, vol. 15, pp. 5293-5310, Mar. 2015.

[9] G. B. Carvalho, S. Theil, and H. K. Kuga, "Imu: Generic model development approach," in Simposio Brasileiro de Engenharia Inercial, pp. 62-67, 2007.

[10] M. E. Parés, J. J. Rosales, and I. Colomina, "Yet another imu simulator: validation and applications," in European Calibration and Orientation Workshop, 2008.

[11] X. Kong, "Ins algorithm using quaternion model for low cost imu," Robotics and Autonomous Systems, vol. 46, pp. 221-246, 2004.

[12] V. Sircoulomb, Etude des concepts de filtrage robuste aux méconnaissances de modèle et aux pertes de mesures. $\mathrm{PhD}$ thesis, Nancy-Université, Nancy, France, Dec. 2008.

[13] P. H. Zipfel, Modeling and Simulation of Aerospace Vehicle Dynamics. American Institute of Aeronautics and Astronautics, second ed., 2000.

[14] J. Radix, Systèmes Inertiels à Composants Liés "Strap-Down". Sup'Aero, Cépaduès-Editions, 1991.

[15] R. Goldman, "Understanding quaternions," Graphical Models, vol. 73, pp. 21-49, Mar. 2011.

[16] Y. Yang, "Spacecraft attitude determination and control: Quaternion based method," Annual Reviews in Control, vol. 36, pp. 198-219, Dec. 2012.

[17] Y. Wu and J. Wang, "Stochastic modeling of inertial errors for mobile mapping applications," in International Symposium on Mobile Mapping Technology, (Tainan, Taiwan), 2013.

[18] A. G. Quinchia, G. Falco, E. Falletti, F. Dovis, and C. Ferrer, "A comparison between different error modeling of mems applied to gps/ins integrated systems," Sensors, vol. 13, no. 8, pp. 9549-9588, 2013.

[19] X. T. B.V., "Mti user manual," Jan. 2014.

[20] N. El-Sheimy, H. Hou, and W. Niu, "Analysis and modeling of inertial sensors using allan variance," IEEE Transactions on Instrumentation and Measurement, vol. 57, pp. 140-149, Jan. 2008. 